\title{
Imágenes en obesidad: contribuyendo a un entendimiento más profundo de la enfermedad
}

Tomás Labbé Atenas ${ }^{1,2, *}$, Constanza Aguilera Arriagada', Juvenal A. Ríos Leal ${ }^{3,4}$, Pía Venegas Araneda', Carolina Neira Ojeda'.

1. Departamento de Enfermedades No Transmisibles, División de Prevención y Control de Enfermedades, Subsecretaría de Salud Pública, Ministerio de Salud. Santiago, Chile.

2. Escuela de Medicina, Facultad de Ciencias Médicas, Universidad de Santiago de Chile. Santiago, Chile.

3. Departamento de Manejo Integral del Cáncer y Otros Tumores, División de Prevención y Control de Enfermedades, Subsecretaría de Salud Pública, Ministerio de Salud. Santiago, Chile.

4. Programas para el futuro. Facultad de Estudios Interdisciplinarios y Escuela de Medicina. Facultad de Ciencias, Universidad Mayor. Santiago, Chile.

\section{Imaging in obesity: contributing to a deeper understanding of the disease}

Resumen: la obesidad constituye un problema mayor de salud pública relevante en la actualidad, considerando que cumple un doble rol al actuar como factor de riesgo para la mayoría de las enfermedades crónicas no transmisibles y constituir una entidad nosológica independiente. Los estudios de imágenes han contribuido, desde diferentes perspectivas, a dilucidar los mecanismos propios de la enfermedad, sus complicaciones, progresión, mecanismos cognitivos involucrados y respuesta a diversos esquemas terapéuticos. El objetivo del presente artículo es proveer de una visión general respecto a cómo los estudios imagenológicos, especialmente basados en resonancia magnética, han profundizado la comprensión de los mecanismos metabólicos y neurocognitivos, relacionados, así como elementos vinculados al tratamiento. Adicionalmente se discuten posibles direcciones futuras en este campo.

Palabras clave: Fisiopatología, Obesidad, Radiología, Resonancia Magnética.

Abstract: Obesity constitutes a relevant issue in public health. It acts both like a risk factor for most of the non-communicable diseases and as an independent nosologic entity. The imaging studies have contributed, from several perspectives, to elucidate the underlying mechanisms of the disease. its complications, progression, involved cognitive phenomena and the response to different therapeutic approaches. The objective of this article is to provide a global view about how the imaging studies, particularly those based on magnetic resonance imaging, have given a deeper comprehension of the related metabolic and cognitive mechanisms and some facts related to the treatment. Additionally, future directions of this field are discussed.

Key word: Magnetic Resonance Imaging, Obesity, Patho, Radiology.

Labbé $T$, et al.Imágenes en obesidad: contribuyendo a un entendimiento más profundo de la enfermedad. Rev Chil Radiol 2020; 26(1): 17-24.

${ }^{*}$ Correo electrónico: Tomás Labbé Atenas MD, PhD. / tomas.labbe@usach.cl

Trabajo enviado el 16 de agosto de 2019. Aceptado para publicación el 18 de marzo de 2020.

\section{Introducción}

La obesidad ha sido históricamente considerada un importante factor de riesgo para diversas patologías de la esfera metabólica y cardiovascular que constituyen las principales causas de muerte para la mayoría de los países desarrollados y en vías de desarrollo en la actualidad. Sin embargo, en la actualidad es abordada cada vez más como una enfermedad y no sólo como un factor de riesgo cardiovascular debido a su complejidad fisiopatológica ${ }^{(1)}$ y a la profunda re- lación que guarda con cambios en un amplio número de sistemas ${ }^{(1,2,3)}$.

Desde la aproximación neurocientífica, existe evidencia de que las personas con obesidad o sobrepeso manifiestan más alteraciones en el dominio de las funciones ejecutivas, hallazgo que a su vez se ha visto asociado a la aparición de diferencias en estudios de neuroimágenes, en comparación con sujetos sanos ${ }^{(4)}$. Así también, conductas poco saludables como el consumo excesivo de azúcar 
se ha relacionado con el compromiso de vías de retroalimentación cerebro-metabólicas, sensibles al efecto de situaciones como estrés ${ }^{(4,5)}$.

Desde el punto de vista metabólico, el exceso del contenido corporal de grasa propio de la obesidad ha sido vinculado a diversos procesos, como niveles inflamatorios periféricos que interactúan con factores genéticos y ambientales para dar cuenta de deterioros orgánicos relacionados con la edad(6). Así mismo, patrones específicos de acumulación de grasa guardan estrecha relación con componentes conductuales como el sedentarismo ${ }^{(6,7)}$, con cambios en la actividad de mediadores séricos relevantes como insulina y adiponectina ${ }^{(8)}$ y con el riesgo de enfermedad aterosclerótica ${ }^{(8,9)}$.

Por otro lado, estudios de imagen han permitido valorar los cambios inducidos por diversos abordajes terapéuticos en obesidad. De esta manera, programas de ejercicio para personas obesas han demostrado cambios en la distribución corporal de grasa y en la sensibilidad periférica al efecto de la insulina ${ }^{(10)}$. Así también, se ha observado que intervenciones más invasivas, como la cirugía bariátrica, generarían cambios en los sistemas cerebrales de recompensa asociados a la alimentación ${ }^{(11)}$ y regresión de alteraciones estructurales propias del impacto de la patología en el sistema cardiovascular ${ }^{(12)}$.

\section{Técnicas de estudio de la composición corporal: Generalidades}

Considerando diversas perspectivas, la obesidad ha sido abordada utilizando otras técnicas de imagen como Tomografía Axial Computada ${ }^{(13)}$ y Ultrasonido ${ }^{(14,15)}$ para la cuantificación de adiposidad abdominal, visceral o subcutánea. Sin embargo, por estar exenta de radiación ionizante y no ser operador-dependiente, hemos limitado la presente revisión a la evidencia derivada de la técnica de resonancia magnética. En contadas ocasiones, literatura complementaria de otras metodologías se menciona con la respectiva aclaración. El objetivo del presente artículo es proveer una visión general de cómo los estudios basados en resonancia magnética han contribuido a la comprensión de los fenómenos metabólicos y neurodinámicos asociados a la patogenia de la obesidad y su tratamiento.

A pesar de que algunas de las técnicas disponibles están fuera del foco principal de interés de este artículo, es importante mencionar que existe una amplia gama de metodologías para el estudio de la composición corporal que pueden contribuir al estudio de pacientes obesos, dependiendo de las cuantificaciones que sean de interés para cada diseño experimental. A continuación, mencionamos de forma sintética otras técnicas que pueden ser utilizadas en el estudio de esta y otras patologías que involucran alteraciones en la composición normal de los tejidos ${ }^{(16,17)}$.

1. Tomografía computada: Permite obtener imágenes tridimensionales de alta resolución. El nivel de atenuación de los rayos $\mathrm{X}$ permite diferenciar los diferentes tejidos, entre ellos la grasa. Diferentes mediciones de interés, como tejido adiposo en hígado o músculo, pueden ser realizadas con esta técnica.

2. Cuantificación de potasio de cuerpo completo: Técnica basada en la distribución fundamentalmente intracelular del potasio en el cuerpo. De esta manera, se cuantifica la masa celular total corporal, estimando a la vez el volumen total de agua, contenido graso, proteínas y músculo. Es particularmente útil en condiciones de hidratación cambiante como embarazo, infancia y malnutrición.

3. Bioimpedanciometría: Ampliamente utilizada en clínica e investigación. A través de la utilización de múltiples frecuencias, en la actualidad es posible estimar el contenido corporal total de agua, así como la proporción de tejido graso y libre de grasa.

4. Absorciometría de Rayos $X$ de Energía Dual: Utiliza energía de rayos $X$. A través de la atenuación de estos $y$, siendo conocidas ciertas características físicas de los tejidos, es posible estimar contenido total de grasa, músculo y tejido óseo mineral.

5. Hidrometría: Basada en la dilución de isótopos administrados al paciente, esta técnica estima la concentración que estos alcanzan en diferentes fluidos, obteniendo estimaciones como la cantidad total de agua corporal.

6. Pletismografía por Desplazamiento de Aire: Obtiene mediciones como la densidad corporal y cuantificaciones de tejido graso y magro. De manera general, estas estimaciones se realizan a partir de los cambios de volumen de aire en una cámara en reposo y cuando el paciente se ha sentado en ella.

7. Hidrodensitometría: Este método mide la cantidad de agua desplazada al sumergir completamente el cuerpo. Considerando factores como el volumen residual pulmonar, se llega a adecuadas estimaciones del volumen y la densidad corporal, así como el porcentaje de grasa corporal

8. EcoMRI: Esta técnica se encuentra en la frontera del desarrollo tecnológico actual. Los valores emanados incluyen el nivel de masa grasa y masa magra. Aunque hasta la fecha es comparativamente menos accesible, exhibe ciertas ventajas como su adecuación y validación para la aplicación en pacientes pediátricos.

9. Espectroscopía: Orientada fundamentalmente a la cuantificación del contenido de lípidos en células hepáticas y musculares. Opera con 
altos niveles de sensibilidad y especificidad planteándose como una alternativa cada vez más potente a la realización de biopsia hepática.

10. Tomografía por emisión de positrones: Considerando que el uso trazador como Fluordesoxiglucosa (FDG) permite la identificación de áreas de alto consumo metabólico y con ello la localización y cuantificación de grasa parda. Los niveles de este tejido han mostrado correlaciones inversas con niveles de IMC y un efecto protector para la aparición de obesidad.

\section{Grasa y metabolismo}

Los estudios de resonancia magnética han permitido, a la fecha, realizar predicciones cada vez más acuciosas del contenido de grasa visceral, con buenos niveles de correlación con estimadores clínicos clásicos como el perímetro de cintura o el índice de masa corporal aunque el nivel de asociación difiere entre hombres y mujeres ${ }^{(18)}$.

Incluso en ausencia de otros factores de riesgo cardiovascular, la presencia de obesidad se ha asociado a diversos procesos y cambios de la esfera metabólica que pueden ser objetivados por técnicas de imagen. De esta forma, elementos específicos del sistema circulatorio como la velocidad del pulso en la pared de la aorta, se ha relacionado con mayor riesgo de eventos cardiovasculares en personas obesas, de forma reversible con la pérdida de peso ${ }^{(19)}$. Además, otras medidas estructurales relacionadas al sistema cardiovascular, como el nivel de tejido adiposo de ubicación pericárdica se han relacionado significativamente con niveles de grasa visceral, hepática y fenómenos como resistencia a la insulina ${ }^{(20)}$.

Desde el punto de vista de la acumulación de grasa visceral, es importante destacar que cuantificaciones estandarizadas a niveles específicos del abdomen han mostrado buenos niveles de asociación con los riesgos de salud cardiovascular en hombres y mujeres. No obstante, la literatura destaca que la estandarización de los niveles y procedimientos para la cuantificación resultan elementos críticos para la validez de estas interpretaciones ${ }^{(21)}$. Aunque existen diversas posibles mediciones en el contexto del estudio imagenológico de la obesidad, cuando la utilidad de la cuantificación del tejido adiposo visceral, subcutáneo e intrahepático han sido comparadas, sólo el volumen de tejido adiposo visceral ha mantenido una asociación independiente, por ejemplo, con la incidencia de hipertensión arterial ${ }^{(22)}$.

En estudios con adolescentes, el uso de metformina en el contexto de síndrome metabólico se ha asociado a una disminución de la adiposidad visceral, fenómeno que a su vez se traduce en mayor sensibilidad al efecto de la insulina ${ }^{(23)}$. Aportes desde otras técnicas de imagen funcional, como Tomografía por Emisión de Positrones han complementado estos resultados mostrando que aunque la insulina y la bradikinina son potentes estimuladores del flujo sanguíneo y gasto metabólico, este efecto se ve atenuado en sujetos obesos con mayor contenido de grasa visceral(23,24). Adicionalmente, la capacidad de activar de forma dirigida el gasto metabólico de la grasa parda se ve conservada en sujetos delgados y comprometido en sujetos obesos ${ }^{(25)}$. Lo anteriormente descrito da cuenta de las condiciones para el establecimiento de un círculo vicioso entre la acumulación de tejido adiposo y la alteración del balance hormonal y energético.

En el sistema osteomuscular, se ha mostrado que la presencia de obesidad no es inocua. Se ha registrado que el depósito de tejido adiposo intramuscular, además de modificar sus características de fuerza y locomoción, se asocia con la aparición de diabetes mellitus y neuropatía periférica ${ }^{(26)}$. Así también, el efecto de la obesidad sobre la integridad estructural del cartílago ha sido sistemáticamente ${ }^{(27)}$.

Aunque la mayoría de la evidencia reportada se refiere al rol metabólico de la grasa visceral general, la medición de grasa intrahepática ha demostrado recientemente que puede constituir un elemento útil en la predicción de la grasa visceral total, los niveles inflamatorios y los trastornos metabólicos de forma independiente( ${ }^{(28,29)}$.

\section{Conducta}

Desde la de las aplicaciones neurocientíficas de la resonancia magnética funcional (RMf) existe un cúmulo importante de evidencia relacionado con los componentes cognitivos de esta patología y las conductas asociadas. A grandes rasgos, se han identificado cambios en la actividad de regiones límbicas y paralímbicas relacionadas con los sistemas de saliencia, así como en regiones prefrontales relacionadas con el control de la conducta en relación a situaciones vinculadas a la ingesta ${ }^{(30)}$.

Los estudios de RMf que son adquiridos durante el reposo, es decir, en ausencia de una prueba o contexto cognitivo que se relacione con la ingesta alimentaria han mostrado que la conectividad funcional de regiones relacionadas con el control cognitivo, la motivación de la conducta y los sistemas de recompensa muestran cambios significativos en personas con obesidad al ser comparadas con la dinámica cerebral basal de personas sanas ${ }^{(31,32)}$.

En paradigmas experimentales que evalúan el funcionamiento de los sistemas de recompensa asociados a la ingesta alimentaria se han identificado menor actividad de regiones como la corteza cingulada y el nucleo caudado en relación a un compromiso de las funciones de control ejecutivo en personas obesas ${ }^{(33)}$. Así también, desde el enfoque de redes neuronales, la red prefrontal ${ }^{(34)} \mathrm{y}$ otros agregados funcionales 
que incluyen a la amígdala, el estriado ventral(35) y el hipotálamo ${ }^{(36)}$, han evidenciado las diferencias en la conectividad cerebral funcional de personas con peso normal y personas con sobrepeso u obesidad.

Otras aproximaciones experimentales han valorado el rol de la integridad del sueño con el buen funcionamiento de los sistemas neuronales responsables de la conducta alimentaria antes mencionados. De esta manera, personas con alteración del sueño expresan cambios en la dinámica de los sistemas neuronales de recompensa y control de la conducta tras la exposición a claves ambientales relacionadas con la comida ${ }^{(37)}$. Es así como personas privadas de sueño de manera aguda o crónica, muestran cambios en la actividad regional de segmentos como la corteza cingulada anterior, el putamen, caudado, tálamo, corteza prefrontal ventromedial e ínsula, favoreciendo el desarrollo de conductas alimentarias poco saludables ${ }^{(38,39)}$

Adicionalmente, alteraciones en la Red por Defecto (Default Mode Network), un agregado funcional con amplia vinculación a diversos procesos cognitivos, ha mostrado sistemáticos cambios en su respuesta funcional cuando personas con obesidad son expuestas a imágenes de comida ${ }^{(40)}$. Estudios bajo la aproximación basada en regiones de interés han mostrado además cambios en la actividad orbitofrontal, e insular frente a estos estímulos, traduciéndose a nivel conductual en una alteración de la experiencia subjetiva de apetito ${ }^{(41)}$.

Otros datos importantes para la comprensión del fenómeno clínico de la obesidad derivan de esta aproximación experimental. Así, los niveles y sensibilidad a la insulina se relacionan con cambios funcionales en las redes involucradas en la conducta alimentaria ${ }^{(42)}$ y la modulación de los sistemas neurocognitivos involucrados tiende a deteriorarse con la edad, explicando parcialmente el aumento de prevalencia de la obesidad en personas mayores ${ }^{(43)}$.

Múltiples tratamientos para el control de la obesidad, tanto convencionales como noveles, han sido abordados desde la perspectiva neurocientífca utilizando la RMf. De esta forma, el efecto farmacológico de fármacos como sibutramina ${ }^{(44)}$, cannabis $^{(45)}$, nicotina $^{(46)}$ y naltrexona ${ }^{(47)}$, han demostrado tener efectos identificables en la dinámica cerebral que se correlacionan en muchos casos con conductas alimentarias y niveles de adiposidad. En el caso de la insulina, se ha observado que induce modificaciones en la actividad cerebral que se ven alteradas en las personas con obesidad, estableciéndose una relación entre esta supuesta "resistencia neuronal" al efecto de la insulina con los niveles de grasa visceral ${ }^{(48)}$. Así mismo, se ha descrito que se requieren altos niveles de sensibilidad periférica a la insulina para que la administración de esta se relacione con cambios en la conectividad de regiones como el hipotálamo, probablemente mediando alteraciones en el fenómeno de la saciedad ${ }^{(49)}$.

\section{Tratamiento}

El tratamiento de la obesidad constituye un desafío importante dado que su existencia reviste diversos riesgos reversibles como el elevado perfil inflamatorio, de relevancia para el contexto quirúrgico ${ }^{(50)} \mathrm{O}$ el riesgo cardiovascular, relacionado con el contenido adiposo visceral, que a su vez es reversible por cambios en el estilo de vida (Pacífico, 2013). En este contexto, tratamientos comunes como el uso de metformina en síndrome metabólico ha demostrado modificar la distribución adiposa y con ello modificar el riesgo global de los pacientes (Srinivasan, 2006). En este segmento nos concentramos en el efecto de dos de las propuestas terapéuticas más validadas y costo efectivas como son las modificaciones de la dieta y el ejercicio físico.

En primer lugar, las intervenciones dietéticas con restricción del aporte calórico han demostrado ${ }^{(51)}$ disminuir los depósitos de grasa intrahepáticos ${ }^{(52)}$. Así también, la restricción del consumo de bebidas azucaradas ha demostrado controlar el depósito de grasa visceral(53). Adicionalmente, programas de educación alimentaria y dietas saludables han demostrado un impacto que excede a la cuantificación del contenido adiposo visceral, llegando a comprobar que podrían influir en la actividad de redes neuronales que se relacionan con el control de la ingesta alimentaria ${ }^{(54)}$. Así también, se ha sugerido recientemente que el consumo de alimentos no procesados como las nueces influye sobre las redes de saliencia y control cognitivo conduciendo a conductas alimentarias beneficiosas desde el punto de vista metabólico(55).

Por otro lado, es amplia la evidencia que da cuenta de los cambios en la composición corporal asociados al ejercicio físico. En esta línea, el ejercicio aeróbico se ha asociado a disminución cuantificable del contenido de grasa visceral y subcutánea ${ }^{(56,57)}$, e incluso en la ausencia de disminución de peso, se ha demostrado la reducción de grasa intrahepática ${ }^{(58,59)}$ y abdominal asociándose a mejora en el perfil metabólico y lipídico ${ }^{(60)}$. Por su parte, el ejercicio de resistencia ha demostrado modificar el contenido de tejido adiposo intramuscular, contribuyendo de forma independiente al control de peso y redistribución corporal.

En el contexto de personas con determinadas patologías crónicas, el efecto del ejercicio también ha sido valorado por técnicas de imagen. De esta manera, en personas con diabetes tipo 2, el ejercicio regular, además de producir pérdida de peso, disminuye sustancialmente el contenido de grasa total y visceral|(61), mejorando además el perfil de sensibilidad a la insulina en forma correlacionada con la modificación de la adiposidad abdominal(62). En otros grupos de pacientes, como aquellos con depresión mayor, 
los programas de ejercicio han demostrado efectividad en disminuir el contenido de grasa pericárdica $y$, con ello, modificando o amortiguando efectos del síndrome metabólico ${ }^{(63)}$. Aunque las asociaciones han sido variables a través de la literatura en cuanto a sus umbrales y su relación con la pérdida de peso, existen algunos datos que vincularían una reducción mínima de 5\% en el Índice de Masa Corporal para generar reducciones significativas, por ejemplo, en el contenido hepático de grasa ${ }^{(64)}$.

Otras medidas terapéuticas, como el uso de pioglitazona, exenatida o rimonabant han demostrado reducciones en el contenido de grasa intrahepático y en el volumen de ateroma respectivamente ${ }^{(65,66,67)}$. Paralelamente, el impacto de la cirugía bariátrica en la reducción del contenido hepático y general de grasa ha sido demostrado a través de resonancia magnéti$\mathrm{ca}^{(68,69)}$. No obstante, lo anterior, estas asociaciones tienen menos consistencia en la literatura y se enfocan en el tratamiento de pacientes con importantes alteraciones metabólicas por sobre la prevención en personas sanas o en riesgo.

\section{Desafíos futuros}

Las técnicas de imagen, específicamente la resonancia magnética, han contribuido de manera importante a la comprensión de los fenómenos anatómicos, fisiopatológicos y neurodinámicos relacionados con la obesidad si bien no existe una única postura respecto a la validación de elementos del examen físico que ayuden a sospechar un nivel riesgoso de adiposidad que se beneficie categóricamente de un estudio imagenológico. Datos recientes han señalado que hasta trece descriptores corporales objetivables clínicamente tendrían una correlación significativa con las cuantificaciones basadas en imagen, exhibiendo perfiles distintos para el tipo de grasa estudiada y características del paciente, como su sexo ${ }^{(70)}$.

El incremento alarmante en la prevalencia de esta patología plantea la necesidad de identificar mecanismos neuronales que favorezcan la adecuada regulación homeostática de la ingesta de alimentos. Esto con el objetivo de buscar qué redes neuronales favorables a la obesidad se conviertan en redes sanas a través de metodologías emergentes como la modulación con fines terapéuticos de las redes neuronales ${ }^{(31,47,71)}$. Así también, moléculas que históricamente han estado vinculadas a otros procesos, como la oxitocina, ofrecen la expectativa de contribuir a modular los procesos cognitivos que dan forma a las conductas alimentarias ${ }^{(72)}$.

Hoy se encuentra pendiente la definición de un estándar transferible en que las cuantificaciones por diferentes aplicaciones de la resonancia magnética puedan contribuir de manera categórica a la estimación de riesgo cardiovascular en personas con sobrepeso y obesidad ${ }^{(73)}$. Estas relaciones estarán determinadas por diversos factores, partiendo por el género, pero constituyen una herramienta útil en la cuantificación de la adiposidad(74).

A pesar de las limitaciones en transferencia y heterogeneidad de los datos aquí analizados, la resonancia magnética ha contribuido con información relevante sobre la fisiopatología de la obesidad, los aspectos neurocognitivos que la perpetúa y el efecto de sus diferentes aproximaciones terapéuticas. Resta el desafío de homogeneizar los métodos y establecer categóricamente el rol de cada medición en la valoración clínica del riesgo de las personas con obesidad.

\section{Referencias}

1. Aguilera C, Labbé T, Busquets J, Venegas P, Neira C, Valenzuela Á. Obesidad: ¿Factor de riesgo o enfermedad? Revista médica de Chile. 2019; 147: 470-474. Available from: http://dx.doi.org/10.4067/ s0034-98872019000400470

2. Kim MK, Reaven GM, Chen Y-DI, Kim E, Kim SH. Hyperinsulinemia in individuals with obesity: Role of insulin clearance. Obesity. 2015; 23: 2430-2434. Available from: http://dx.doi.org/10.1002/oby.21256

3. Pacheco-Cervera J, Codoñer-Franch P, Simó-Jordá R, Pons-Vázquez S, Galbis-Estrada C, Pinazo-Durán MD. Reduced retinal nerve fibre layer thickness in children with severe obesity. Pediatric Obesity. 2015; 10: 448-453. Available from: http://dx.doi.org/10.1111/ ijpo.12005

4. Bora E, McIntyre RS, Ozerdem A. Neurococognitive and neuroimaging correlates of obesity and components of metabolic syndrome in bipolar disorder: a systematic review. Psychol Med. 2019 apr; 49(5): 738-749.

5. Tryon MS, Stanhope KL, Epel ES, Mason AE, Brown $R$, Medici V, et al. Excessive Sugar Consumption May Be a Difficult Habit to Break: A View From the Brain and Body. J Clin Endocrinol Metab. 2015 jun; 100(6): 2239-2247.

6. Corlier F, Hafzalla G, Faskowitz J, Kuller LH, Becker JT, Lopez OL, et al. Systemic inflammation as a predictor of brain aging: Contributions of physical activity, metabolic risk, and genetic risk. Neuroimage. 2018 may 15; 172: 118-129.

7. Henson J, Edwardson CL, Morgan B, Horsfield MA, Khunti K, Davies MJ, et al. Sedentary time and MRIderived measures of adiposity in active versus inactive individuals. Obesity. 2018 jan; 26(1): 29-36.

8. Huang GZ, Tang YH, Wang BY, Zhang B, Hu TJ, Zhang $L$, et al. Effects of telmisartan on insulin resistance and visceral fat distribution in Chinese hypertensive patients with obesity. Saudi Med J. 2011 oct; 32(10): 1017-1021.

9. Frühbeck G, Catalán V, Rodríguez A, Gómez-Ambrosi J. Adiponectin-leptin ratio: A promising index to estimate adipose tissue dysfunction. Relation with obesityassociated cardiometabolic risk. Adipocyte. 2018 jan 2; 7(1): 57-62.

10. Reichkendler MH, Auerbach P, Rosenkilde M, Christensen AN, Holm S, Petersen MB, et al. Exercise training favors increased insulin-stimulated glucose uptake in skeletal muscle in contrast to adipose tissue: a randomized study using FDG PET imaging. Am J 
Physiol Endocrinol Metab. 2013 aug 15; 05(4): E496506.

11. Goldstone AP, Miras AD, Scholtz S, Jackson S, Neff KJ, Pénicaud L, et al. Link Between Increased Satiety Gut Hormones and Reduced Food Reward After Gastric Bypass Surgery for Obesity. J Clin Endocrinol Metab. 2016 feb; 101(2): 599-609.

12. Cuspidi C, Tadic M, Grassi G. Diastolic dysfunction, blood pressure and obesity: new insights from a general population. J Hypertens. 2014 dec; 32(12): 2359-2361.

13. Sebro R. Obesity, hepatic steatosis, and their impact on fat Infiltration of the trunk musculature using Unenhanced Computed Tomography. Journal of Computer Assisted Tomography. 2017; 41: 298-301. Available from: http://dx.doi.org/10.1097/rct.0000000000000507

14. Rolfe EDL, De Lucia Rolfe E, Sleigh A, Finucane FM, Brage S, Stolk RP, et al. Ultrasound measurements of visceral and subcutaneous abdominal thickness to predict abdominal adiposity among older men and women. Obesity. 2010; 18: 625-631. Available from: http://dx.doi.org/10.1038/oby.2009.309

15. Rolfe EDL, De Lucia Rolfe E, Modi N, Uthaya S, Hughes IA, Dunger DB, et al. Ultrasound estimates of visceral and subcutaneous-abdominal adipose tissues in infancy. Journal of Obesity. 2013; 1-9. Available from: http://dx.doi.org/10.1155/2013/951954

16. Kuriyan R. Body composition techniques. Indian J Med Res. 2018 nov; 148(5): 648-658.

17. Lemos T, Gallagher D. Current body composition measurement techniques. Curr Opin Endocrinol Diabetes Obes. 2017 oct; 24(5): 310-314

18. Barreira TV, Broyles ST, Gupta AK, Katzmarzyk PT. Relationship of anthropometric indices to abdominal and total body fat in youth: sex and race differences. Obesity. 2014 may; 22(5): 1345-1350.

19. Rider OJ, Tayal U, Francis JM, Ali MK, Robinson MR, Byrne JP, et al. The effect of obesity and weight loss on aortic pulse wave velocity as assessed by magnetic resonance imaging. Obesity. $2010 \mathrm{dec}$; 18(12): 23112316.

20. Bosy-Westphal A, Kossel E, Goele K, Blöcker T, Lagerpusch M, Later W, et al. Association of pericardial fat with liver fat and insulin sensitivity after diet-induced weight loss in overweight women. Obesity. 2010; 18: 2111-2117. Available from: http://dx.doi.org/10.1038/ oby. 2010.49

21. Shen W, Punyanitya M, Chen J, Gallagher D, Albu J, Pi-Sunyer $\mathrm{X}$, et al. Visceral adipose tissue: relationships between single slice areas at different locations and obesity-related health risks. Int J Obes. 2007 may; 31(5): 763-769.

22. Chandra A, Neeland IJ, Berry JD, Ayers CR, Rohatgi $A$, Das SR, et al. The relationship of body mass and fat distribution with incident hypertension: observations from the Dallas Heart Study. J Am Coll Cardiol. 2014 sep 9; 64(10): 997-1002.

23. Díaz M, Bassols J, López-Bermejo A, de Zegher F, Ibáñez L. Metformin treatment to reduce central adiposity after prenatal growth restraint: a placebocontrolled pilot study in prepubertal children. Pediatr Diabetes. 2015 nov; 16(7): 538-545.

24. Iozzo P, Guiducci L, Guzzardi MA, Pagotto U. Brain PET imaging in obesity and food addiction: current evidence and hypothesis. Obes Facts. 2012 apr 19;
5(2): 155-164.

25. Carey AL, Kingwell BA. Brown adipose tissue in humans: therapeutic potential to combat obesity. Pharmacol Ther. 2013 oct; 140(1): 26-33.

26. Hilton TN, Tuttle LJ, Bohnert KL, Mueller MJ, Sinacore DR. Excessive adipose tissue infiltration in skeletal muscle in individuals with obesity, diabetes mellitus, and peripheral neuropathy: association with performance and function. Phys Ther. 2008 nov; 88(11): 336-344.

27. Mezhov V, Ciccutini FM, Hanna FS, Brennan SL, Wang YY, Urquhart DM, et al. Does obesity affect knee cartilage? A systematic review of magnetic resonance imaging data. Obes Rev. 2014 feb; 15(2): 143-157.

28. Rankin KC, O'Brien LC, Segal L, Khan MR, Gorgey AS. Liver Adiposity and Metabolic Profile in Individuals with Chronic Spinal Cord Injury. Biomed Res Int. 2017 aug 30; 2017: 1364818.

29. Meir AY, Yaskolka Meir A. Intrahepatic fat, abdominal adipose tissues, and metabolic state; magnetic resonance imaging study. Clinical Nutrition. 2018; 37: S7. Available from: http://dx.doi.org/10.1016/j.clnu.2018.06.1094

30. García-García I, Narberhaus A, Marqués-Iturria I, Garolera M, Rădoi A, Segura B, et al. Neural responses to visual food cues: insights from functional magnetic resonance imaging. Eur Eat Disord Rev. 2013 mar; 21(2): 89-98.

31. Lips MA, Wijngaarden MA, van der Grond J, van Buchem MA, de Groot GH, Rombouts SARB, et al. Resting-state functional connectivity of brain regions involved in cognitive control, motivation, and reward is enhanced in obese females. Am J Clin Nutr. 2014 aug; 100(2): 524-531.

32. Hogenkamp PS, Zhou W, Dahlberg LS, Stark J, Larsen $A L$, Olivo $G$, et al. Higher resting-state activity in reward-related brain circuits in obese versus normalweight females independent of food intake. Int J Obes. 2016 nov; 40(11): 1687-1692.

33. Tuulari JJ, Karlsson HK, Hirvonen J, Salminen P, Nuutila P, Nummenmaa L. Neural circuits for cognitive appetite control in healthy and obese individuals: an fMRI study. PLoS One. 2015 feb 6; 10(2): e0116640.

34. Frank S, Heinze JM, Fritsche A, Linder K, von Feilitzsch M, Königsrainer A, et al. Neuronal Food Reward Activity in Patients With Type 2 Diabetes With Improved Glycemic Control After Bariatric Surgery. Diabetes Care. 2016 aug; 39(8): 1311-1317.

35. Beaver JD, Lawrence AD, van Ditzhuijzen J, Davis $\mathrm{MH}$, Woods A, Calder AJ. Individual differences in reward drive predict neural responses to images of food. J Neurosci. 2006 may 10; 26(19): 5160-5166.

36. Kilpatrick LA, Coveleskie K, Connolly L, Labus JS, Ebrat B, Stains J, et al. Influence of sucrose ingestion on brainstem and hypothalamic intrinsic oscillations in lean and obese women. Gastroenterology. 2014 may; 146(5): 1212-1221.

37. Demos KE, Sweet LH, Hart CN, McCaffery JM, Williams SE, Mailloux KA, et al. The effects of experimental manipulation of sleep duration on neural response to food cues. Sleep. 2017 nov 1; 40(11). Available from: http://dx.doi.org/10.1093/sleep/zsx125

38. Benedict $C$, Brooks SJ, O’Daly OG, Almèn MS, Morell A, Åberg K, et al. Acute sleep deprivation enhances the brain's response to hedonic food stimuli: an fMRI study. J Clin Endocrinol Metab. 2012 mar; 97(3): E443- 


\section{E447.}

39. St-Onge M-P, O'Keeffe M, Roberts AL, RoyChoudhury $A$, Laferrère B. Short sleep duration, glucose dysregulation and hormonal regulation of appetite in men and women. Sleep. 2012 nov 1; 35(11): 1503-1510.

40. Tregellas JR, Wylie KP, Rojas DC, Tanabe J, Martin $\mathrm{J}$, Kronberg E, et al. Altered default network activity in obesity. Obesity. 2011 dec; 19(12): 2316-2321.

41. Porubská K, Veit R, Preissl H, Fritsche A, Birbaumer $N$. Subjective feeling of appetite modulates brain activity: an fMRI study. Neuroimage. 2006 sep; 32(3): 1273-1280.

42. Wallner-Liebmann S, Koschutnig K, Reishofer G, Sorantin E, Blaschitz B, Kruschitz R, et al. Insulin and Hippocampus Activation in Response to Images of High-Calorie Food in Normal Weight and Obese Adolescents. Obesity. 2010; 18: 1552-1557. Available from: http://dx.doi.org/10.1038/oby.2010.26

43. Cheah YS, Lee S, Ashoor G, Nathan Y, Reed LJ, Zelaya FO, et al. Ageing diminishes the modulation of human brain responses to visual food cues by meal ingestion. Int J Obes. 2014 sep; 38(9): 1186-1192.

44. Oltmanns KM, Heldmann M, Daul S, Klose S, Rotte M, Schäfer M, et al. Sibutramine promotes amygdala activity under fasting conditions in obese women. Psychopharmacology. 2012 jun; 221(4): 693-700.

45. Muniyappa R, Sable S, Ouwerkerk R, Mari A, Gharib AM, Walter M, et al. Metabolic effects of chronic cannabis smoking. Diabetes Care. 2013 aug; 36(8): 2415-2422.

46. Kroemer NB, Guevara A, Vollstädt-Klein S, Smolka MN. Nicotine alters food-cue reactivity via networks extending from the hypothalamus. Neuropsychopharmacology. 2013 oct; 38(11): 2307-2314.

47. Murray E, Brouwer S, McCutcheon R, Harmer CJ, Cowen PJ, McCabe C. Opposing neural effects of naltrexone on food reward and aversion: implications for the treatment of obesity. Psychopharmacology. 2014 nov; 231(22): 4323-4335.

48. Kullmann S, Heni M, Veit R, Scheffler K, Machann $\mathrm{J}$, Häring $\mathrm{H}-\mathrm{U}$, et al. Selective insulin resistance in homeostatic and cognitive control brain areas in overweight and obese adults. Diabetes Care. 2015 jun; 38(6): 1044-1050.

49. Kullmann S, Heni M, Veit R, Scheffler K, Machann J, Häring $\mathrm{H}-\mathrm{U}$, et al. Intranasal insulin enhances brain functional connectivity mediating the relationship between adiposity and subjective feeling of hunger. Sci Rep. 2017 May 9; 7(1): 1627.

50. Holt DQ, Moore GT, Strauss BJG, Hamilton AL, De Cruz $P$, Kamm MA. Visceral adiposity predicts post-operative Crohn's disease recurrence. Aliment Pharmacol Ther. 2017 may; 45(9): 1255-1264.

51. Pacifico L, Arca M, Anania C, Cantisani V, Di Martino $\mathrm{M}$, Chiesa C. Arterial function and structure after a 1-year lifestyle intervention in children with nonalcoholic fatty liver disease. Nutr Metab Cardiovasc Dis. 2013 oct; 23(10): 1010-1016.

52. Haufe S, Haas V, Utz W, Birkenfeld AL, Jeran S, Böhnke $\mathrm{J}$, et al. Long-lasting improvements in liver fat and metabolism despite body weight regain after dietary weight loss. Diabetes Care. 2013 nov; 36(11): 3786-3792.

53. Maersk M, Belza A, Stødkilde-Jørgensen H, Ringgaard
S, Chabanova E, Thomsen $\mathrm{H}$, et al. Sucrose-sweetened beverages increase fat storage in the liver, muscle, and visceral fat depot: a 6 -mo randomized intervention study. Am J Clin Nutr. 2012 feb; 95(2): 283-289.

54. Kahathuduwa CN, Davis T, O'Boyle M, Boyd LA, Chin $\mathrm{S}-\mathrm{H}$, Paniukov D, et al. Effects of 3-week total meal replacement vs. typical food-based diet on human brain functional magnetic resonance imaging foodcue reactivity and functional connectivity in people with obesity. Appetite. 2018 jan 1; 120: 431-441.

55. Farr OM, Tuccinardi D, Upadhyay J, Oussaada SM, Mantzoros CS. Walnut consumption increases activation of the insula to highly desirable food cues: A randomized, double-blind, placebo-controlled, crossover fMRI study. Diabetes Obes Metab. 2018 jan; 20(1): 173-177.

56. Alberga AS, Farnesi B-C, Lafleche A, Legault L, Komorowski $\mathrm{J}$. The effects of resistance exercise training on body composition and strength in obese prepubertal children. Phys Sportsmed. 2013 sep; 41(3): 103-109.

57. Ross R, Rissanen J. Mobilization of visceral and subcutaneous adipose tissue in response to energy restriction and exercise. Am J Clin Nutr. 1994 nov; 60(5): 695-703.

58. Johnson NA, Sachinwalla T, Walton DW, Smith K, Armstrong A, Thompson MW, et al. Aerobic exercise training reduces hepatic and visceral lipids in obese individuals without weight loss. Hepatology. 2009 oct; 50(4): 1105-1112.

59. Lee J, Lee S, Shin S, Kang HS. Association between the GNB3 Polymorphism and Blood Pressure in Young Korean Men. Medicine \& Science in Sports \& Exercise. 2005; 37: 1138-1143. Available from: http:// dx.doi.org/10.1249/01.mss.0000170096.96662.05

60. Meir AY, Shelef I, Schwarzfuchs D, Gepner Y, Tene $\mathrm{L}$, Zelicha $\mathrm{H}$, et al. Intermuscular adipose tissue and thigh muscle area dynamics during an 18-month randomized weight loss trial. J Appl Physiol. 2016; 21: 518-527. Available from: http://dx.doi.org/10.1152/ japplphysiol.00309.2016

61. Lee S, Kuk JL, Davidson LE, Hudson R, Kilpatrick $\mathrm{K}$, Graham TE, et al. Exercise without weight loss is an effective strategy for obesity reduction in obese individuals with and without Type 2 diabetes. J Appl Physiol. 2005 Sep; 99(3): 1220-1225.

62. Lee S, Bacha F, Hannon T, Kuk JL, Boesch C, Arslanian S. Effects of aerobic versus resistance exercise without caloric restriction on abdominal fat, intrahepatic lipid, and insulin sensitivity in obese adolescent boys: a randomized, controlled trial. Diabetes. 2012 Nov; 61(11): 2787-2795.

63. Kahl KG, Kerling A, Tegtbur U, Gützlaff E, Herrmann $J$, Borchert L, et al. Effects of additional exercise training on epicardial, intra-abdominal and subcutaneous adipose tissue in major depressive disorder: $\mathrm{A}$ randomized pilot study. J Affect Disord. 2016 mar 1; 192: 91-97.

64. Patel NS, Doycheva I, Peterson MR, Hooker J, Kisselva T, Schnabl B, et al. Effect of weight loss on magnetic resonance imaging estimation of liver fat and volume in patients with nonalcoholic steatohepatitis. Clin Gastroenterol Hepatol. 2015 Mar; 13(3): 561-568.e1.

65. Zib I, Jacob AN, Lingvay I, Salinas K, McGavock JM, Raskin $\mathrm{P}$, et al. Effect of pioglitazone therapy on myo- 
cardial and hepatic steatosis in insulin-treated patients with type 2 diabetes. J Investig Med. 2007 jul; 55(5): 230-236.

66. Nissen SE, Nicholls SJ, Wolski K, Rodés-Cabau J, Cannon CP, Deanfield JE, et al. Effect of rimonabant on progression of atherosclerosis in patients with abdominal obesity and coronary artery disease: the STRADIVARIUS randomized controlled trial. JAMA. 2008 apr 2; 299(13): 1547-1560.

67. Dutour A, Abdesselam I, Ancel P, Kober F, Mrad G, Darmon $P$, et al. Exenatide decreases liver fat content and epicardial adipose tissue in patients with obesity and type 2 diabetes: a prospective randomized clinical trial using magnetic resonance imaging and spectroscopy. Diabetes Obes Metab. 2016 sep; 18(9): 882-891.

68. Folini L, Veronelli A, Benetti A, Pozzato C, Cappelletti M, Masci E, et al. Liver steatosis (LS) evaluated through chemical-shift magnetic resonance imaging liver enzymes in morbid obesity; effect of weight loss obtained with intragastric balloon gastric banding. Acta Diabetol. 2014; 51(3): 361-368.

69. Yu H, Di J, Bao Y, Zhang P, Zhang L, Tu Y, et al. Visceral fat area as a new predictor of short-term diabetes remission after Roux-en-Y gastric bypass surgery in Chinese patients with a body mass index less than 35 kg/m2. Surg Obes Relat Dis. 2015 jan; 11(1): 6-11.
70. Sun J, Xu B, Lee J, Freeland-Graves JH. Novel Body Shape Descriptors for Abdominal Adiposity Prediction Using Magnetic Resonance Images and Stereovision Body Images. Obesity. 2017 oct; 25(10): 1795-1801.

71. Winter SR, Yokum S, Stice E, Osipowicz K, Lowe MR. Elevated reward response to receipt of palatable food predicts future weight variability in healthy-weight adolescents. Am J Clin Nutr. 2017 apr; 105(4): 781-789.

72. Striepens N, Schröter F, Stoffel-Wagner B, Maier W, Hurlemann R, Scheele D. Oxytocin enhances cognitive control of food craving in women. Hum Brain Mapp. 2016 dec; 37(12): 4276-4285.

73. Tyagi A, Yeganeh O, Levin Y, Hooker JC, Hamilton GC, Wolfson T, et al. Intra- and inter-examination repeatability of magnetic resonance spectroscopy, magnitude-based MRI, and complex-based MRI for estimation of hepatic proton density fat fraction in overweight and obese children and adults. Abdom Imaging. 2015 oct; 40(8): 3070-3077.

74. Al-Radaideh A, Tayyem R, Al-Fayomi K, Nimer_N, Malkawi A, Al-Zu'bi R, et al. Assessment of Abdominal Fat Using High-field_Magnetic Resonance Imaging and Anthropometric and Biochemical Parameters. The American Journal of the_Medical Sciences. 2016; 352: 593-602. Available from: http://dx.doi.org/10.1016/j. amjms.2016.09.009 